\title{
Scalable Distributed DNN Training using TensorFlow and CUDA-Aware MPI: Characterization, Designs, and Performance Evaluation
}

\author{
Ammar Ahmad Awan, Ching-Hsiang Chu, \\ Hari Subramoni, and Dhabaleswar K. Panda \\ Department of Computer Science and Engineering \\ The Ohio State University \\ \{awan.10, chu.368, subramoni.1, panda.2\}@osu.edu
}

\author{
Jeroen Bédorf \\ Minds.ai \\ Santa Cruz, the United States \\ jeroen@minds.ai \\ Leiden Observatory, Leiden University \\ Leiden, the Netherlands
}

\begin{abstract}
The current wave of advances in Machine Learning (ML) and Deep Learning (DL) have been triggered by the availability of large-scale datasets, efficient CPU and GPU hardware, and development of easy-to-use software frameworks like TensorFlow (TF), Caffe and Torch. TensorFlow has been, by far, the most widely adopted ML/DL framework. However, little exists in the literature that provides a thorough understanding of the capabilities which TensorFlow offers for the distributed training of large ML/DL models that need computation and communication at scale. Most commonly used distributed training approaches for TF can be categorized as follows: 1) Google Remote Procedure Call (gRPC), 2) gRPC+' $X$ ': $X$ = (InfiniBand Verbs, Message Passing Interface (MPI), and GPUDirect RDMA), and 3) No-gRPC: Baidu Allreduce with MPI, Horovod with MPI, and Horovod with NVIDIA NCCL. In this paper, we provide an in-depth performance characterization and analysis of these distributed training approaches on various GPU clusters including the Piz Daint system (\#6 on Top500). We perform experiments to gain novel insights along the following vectors: 1) Application-level scalability of DNN training, 2) Effect of Batch Size on scaling efficiency, 3) Impact of the MPI library used for no-gRPC approaches, and 4) Type and size of DNN architectures (e.g ResNet vs. MobileNet). Based on these experiments, we present two key insights: 1) Overall, No-gRPC designs achieve better performance compared to gRPC-based approaches for most configurations, and 2) The performance of No-gRPC is heavily influenced by the gradient aggregation using the Allreduce communication pattern. Finally, we propose a truly CUDAAware MPI_Allreduce design that exploits 1) CUDA kernels to perform large reductions on the GPU and 2) A pointer cache to avoid overheads involved in queries to the CUDA driver. Our proposed designs have been implemented in MVAPICH2-GDR and offer 5-17 $\times$ better performance than NCCL2 for small and medium messages, and reduces latency by $29 \%$ for large messages on 16 GPUs (nodes). The proposed optimizations help HorovodMPI to achieve approximately $90 \%$ scaling efficiency for ResNet50 training on 64 GPUs. Further, Horovod-MPI achieves $1.8 \times$ and 3.2 $\times$ higher throughput than the native gRPC method for ResNet-50 and MobileNet, respectively, on the Piz Daint cluster.
\end{abstract}

\section{INTRODUCTION}

Deep Learning (DL) has been a significant contributor to the recent achievements in the Artificial Intelligence (AI) realm. Novel approaches like back-propagation in Deep Neural Networks (DNNs) were investigated around the 1980 time frame [36]. However, the potential of these approaches was marred by slow hardware and lack of sufficient training data.
To this end, the current resurgence and a renewed interest in DL and DNN-based solutions to classical as well as new Machine Learning (ML) problems can be attributed to the widespread availability of 1) versatile and large-scale datasets like ImageNet [1], and 2) efficient computation capabilities in modern hardware architectures like Graphics Processing Units (GPUs) and multi-/many-core CPUs. These two trends have positively influenced the development of several high-level DL toolkits like Caffe [9], [25], Microsoft Cognitive Toolkit (CNTK), Facebook PyTorch [5], and Google TensorFlow [11]. Implementing DNN and back-propagation techniques in an efficient manner has been a challenging problem. However, these toolkits have played a crucial role in making ML and DL more accessible for both academic as well as industrybased researchers in various fields. In the context of DL frameworks, it is pertinent to mention that TensorFlow is the most popular DL framework and has seen widespread adoption. Today, more than 1,600 people have contributed to the TensorFlow GitHub repository [6] and several hundred research papers have utilized TensorFlow for both research and commercial applications. However, TensorFlow in its early days was criticized for its slower performance [39] as well as lack of support for efficient distributed training and limited support for High Performance Computing (HPC) systems. To this end, recent efforts by the TF developers as well as the open source community are commendable and performance has significantly improved for both single-GPU/single-node as well as multi-GPU/multi-node (distributed) training. The gRPC [19] library, which is the official distributed training infrastructure for $\mathrm{TF}$, has been optimized for tensor transfers (fewer memory operations), but still uses the relatively slow standard Ethernet networks. However, gRPC can take advantage of InfiniBand (IB) using the IP over IB (IPoIB) protocol which offers significantly better performance. At the same time, the community has been actively exploring Message Passing Interface (MPI) - a de facto standard for the HPC community - based designs to improve distributed training on HPC clusters. However, the active interest and contributions from the community have led to several disjoint efforts and fragmentation in the way users can take advantage of the advanced distributed training designs in TF. The two broad 
challenges that we investigate in this paper are: "1) What is the most efficient tensor communication (for gradient aggregation) framework for TensorFlow and 2) How can we improve the performance of this communication using CUDA-Aware MPI? Several detailed questions follow this broad challenge. It is pertinent to note that little in existing literature can offer insights to the following key questions, which are of significant interest if large-scale distributed training is employed.

- What are the available choices for distributed training using TensorFlow for a given execution platform?

- What are the key features and performance characteristics of the various distributed training approaches for TensorFlow?

- How can we optimize the performance of distributed training using TensorFlow on modern HPC systems?

\section{A. Contributions}

To the best of our knowledge, this is the first paper that offers a comprehensive landscape that highlights, evaluates, and optimizes a diverse set of approaches that deal with distributed DNN training using TensorFlow at scale. In this paper, we make the following key contributions:

- We provide an in-depth categorization, design analysis, and performance characterization of distributed training approaches for TensorFlow using state-of-the-art DNNs like ResNet-50, MobileNet, and NASNet.

- We propose a truly CUDA-Aware MPI_Allreduce design that exploits 1) CUDA kernels to perform large reductions on the GPU and 2) A pointer cache to avoid overheads involved in queries to the CUDA driver.

- We illustrate benefits of the proposed MPI_Allreduce optimizations using micro-benchmarks as well as application workloads (tf_cnn_benchmarks) using TensorFlow and Horovod.

- We present a comprehensive and large-scale performance evaluation (up to 128 GPUs) for all the available distributed training approaches on three modern HPC systems.

\section{OVERVIEW OF COMMUNICATION LIBRARIES FOR}

\section{Distributed Training: Past, Present, and Future}

We first provide a brief historical perspective on various DL frameworks and how they utilize communication libraries for distributed training. Next, we describe communication libraries for distributed training using TensorFlow. We conclude this section with a discussion on how TensorFlow is moving towards a different system to handle community contributions and how it may affect the distributed training approaches we discuss in Section III

\section{A. DL Frameworks and Communication Libraries}

Most ML/DL frameworks started with single-node/singleGPU designs. Caffe, for example, had no support for multinode distributed training and only external efforts [8], [12], [24] provide such support. However, the exponential growth in the size of DNN architectures and an ever-increasing need for speed has forced the ML/DL framework designers to rethink their strategy and to start utilizing existing communication schemes or design their own libraries from scratch. Microsoft Cognitive Toolkit (CNTK) [29] is based on an MPI design whereas Caffe2 [9] uses the Gloo [10] collective communication library developed by Facebook, which is similar to NVIDIA's NCCL [32] library. Gloo exploits the InfiniBand verbs interface and Remote Direct Memory Access (RDMA) technology to offer various reduction algorithms. Apart from the communication libraries that come integrated with the frameworks, there are external (commercial) efforts to enable efficient distributed training. For example, IBM has developed the PowerAI Distributed Deep-learning Library (DDL), which uses a multi-ring reduction algorithm. The library is built on top of IBM's Spectrum MPI (a derivative of OpenMPI) and as such supports all the network interfaces that are supported by the OpenMPI library. According to IBM, the library can be integrated with TensorFlow, Caffe and Torch. Intel has developed the Intel Machine Learning Scaling Library (MLSL) [40]. This library is built on top of Intel MPI and therefore supports various interconnects such as InfiniBand, Omni-Path, and Ethernet. The library offers a set of communication primitives that neural network frameworks can take advantage of when performing distributed communication. According to the research paper it is integrated with Intel-Caffe, TensorFlow, and Intel's own neural-net compiler called nGraph. To bring support to TensorFlow, the library modifies the Horovod [37] library by inserting the MLSL communication primitives.

\section{B. Communication Libraries for TensorFlow}

Our focus in this paper is on distributed training using TensorFlow, which, by default, can be realized using the official Google RPC (gRPC) library. gRPC is a generic pointto-point communication library and has no collective communication support. It works in a simple client/server style fashion and has been tightly integrated with TF. However, TF has been extended by contributors to take advantage of additional communication libraries like MPI, RDMA Verbs, and NCCL. We discuss the libraries that TF uses in more detail in the following sections. $\mathbf{R R P C}$ is a point-to-point RPC library which exchanges data using a pre-defined message format described in the protobuf language [4]. Each node launches a server process responsible for receiving messages from the other nodes. At the launch of TF, the user has to specify IP and port number of the listening server for all the other nodes in the training run. gRPC offers no CUDA-Aware operations, and as such, all data is first staged on the host before being sent over the network. Recent optimizations have however reduced the number of copy operations required to convert GPU buffers into protobuf messages. All the communication operations are handled by a group of threads which allow overlapping data transfers for optimal performance. gRPC uses the HTTP/2 protocol on top of TCP/IP based networks. The generality of the library causes it to also be widely used outside of the deep learning application area. Message Passing Interface (MPI) is a de facto standard for 
expressing distributed-memory programs. Several implementations of the MPI standard like MPICH [2], MVAPICH [30], OpenMPI [42], and CrayMPI [35] have been developed and optimized over the period of several years for various processor architectures and high-performance interconnects like High-speed Ethernet (HSE) and InfiniBand. In recent years, accelerators like NVIDIA GPUs have been adopted by most HPC systems [28]. As a result, MPI extensions have been proposed to support efficient communication between GPUs. Initially, without the capability of direct access of GPU memory, MPI applications required explicit copying of GPU data to a staging buffer in host memory in order to push the data to the network. Similarly, data movement from CPU to GPU was needed after receiving the data on the host through an MPI_Recv operation. This significantly impacts performance as well as productivity. Thus, several MPI libraries including OpenMPI and MVAPICH2-GDR [30] provide CUDA-Aware MPI primitives to transparently perform such copy operations. These CUDA-Aware MPI libraries significantly improve performance and productivity for MPI+CUDA applications. NVIDIA NCCL offers multi-GPU communication primitives. NCCL (pronounced Nickel) has been developed by NVIDIA to efficiently tackle communication for DL workloads. NCCL's API closely resembles the MPI interface and provides communication primitives for broadcast, all-gather, reduce, reducescatter, and all-reduce. Precisely, NCCL's goal is to provide fast communication of messages between GPUs in dense multi-GPU machines like the DGX-1 and DGX-2 [31]. NCCL 1.x was, in fact, a single-node library. However, since its introduction, NCCL has significantly evolved and now offers multi-node collective operations using IB verbs. To launch NCCL2 applications, users still need to rely on an out-ofband mechanism for connection management. MPI launchers like "mpirun" are used to set up connections and assign ranks while NCCL primitives are used for the actual communication.

\section{Future TensorFlow Developments}

Google is currently redesigning how contributions are included in the TensorFlow source tree. When TensorFlow 2.0 is released, a number of options that we have analyzed in this paper might not be available anymore. However, Google understands the importance of supporting multiple networking options and is actively working on creating a special interest group (SIG) related to networking. At the moment of writing, the APIs to add additional communication methods to TF have not been formalized. However, there are plans to reenable the current contributions and introduce support for new communication APIs such as UCX [7].

\section{Analysis of Design and Performance: Several Distributed TRAINING APPROACHES FOR TENSORFLOW}

We now dive into the various distributed training designs for TensorFlow and group them into categories based on how the communication libraries are exploited. Next, we provide the performance analysis for all of these approaches. A high-level hierarchy of all these TF approaches is illustrated in Figure 1

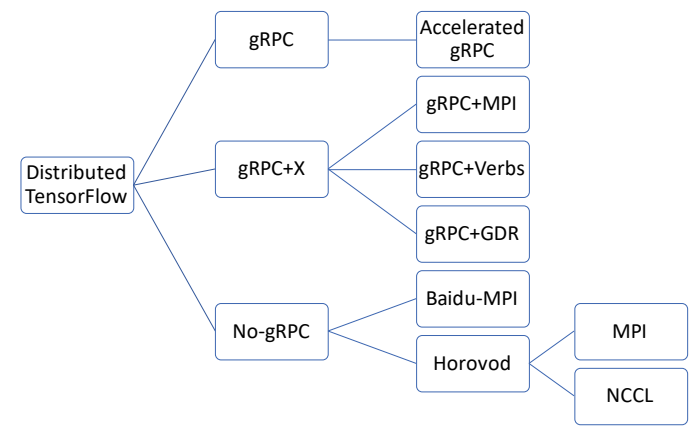

Fig. 1. Various Approaches for Distributed Training using TensorFlow

\section{A. gRPC-based Designs}

The standard distributed training method for TensorFlow is the so-called Parameter Server (PS) model [26]. In this model, there are worker processes that perform the heavy compute work and separate parameter server processes responsible for combining the worker processes results. In other words, the PSs are responsible for storing and updating the parameters (e.g., weights) of the neural network. TensorFlow programs can specify the number of workers and number of PS tasks that should be used. The PS tasks generally do not require much computing power and can run on nodes without accelerators. Depending on the nodes used it is possible to run both a worker process and a PS process on the same machine. TensorFlow comes with a set of support classes that are built on top of the worker and PS instances to enable monitoring and restarting the training process. This can be used for checkpointing (saving) the training state or for fault tolerance in case a worker node crashes due to hardware failure. TensorFlow's PS model utilizes the gRPC library for communication between processes. It allows programs (optionally written in different programming languages) to communicate via the TCP/IP protocol by using RPCs and uniform data buffers. However, the user is responsible for configuring the end-points for each of the launched processes. This can be a labor-intensive task and requires knowledge about the cluster and used nodes. TensorFlow uses the following steps to exchange tensor buffers between the processes: The producer process:

1) After a tensor, for which it has been determined that it has to be sent to another process, has been computed it is placed on a table.

2) If there is no outstanding request for the tensor then it stays in the table until a request is received.

3) If there is a request for this tensor then it is served immediately and removed from the waiting table.

The consumer process:

1) Send a tensor request to the producer.

2) Wait until the producer returns the requested data.

3) Once the data has been received continue processing of the tensor graph

This is an example of a pull model where the data is pulled from producer to consumer. The gRPC library will send the 
tensor request message and the return message that contains the tensor data. This data is encoded via the protobuffer library [4]. If the tensor was computed on the GPU, then the tensor data first has to be completely copied to the host memory before it can be encoded in the protobuffer format and transferred. If the tensor's destination is in GPU memory, then similar steps will have to be taken, where the decoding takes place in the host memory before the decoded data is copied to the GPU. Challenges in Extending gRPC-based

Designs: The tight integration of TF with gRPC makes it non-trivial to add support for additional network protocols. However, TensorFlow's design allows for offloading just the tensor transfers, which are the most data-intensive communication operations during the processing of neural networks. With the high-performance GPUs (and other accelerators) currently available it is critical that the data transfers happen as fast as possible, otherwise, the GPUs would sit idle waiting for data. The more administrative communication operations such as setting up the network and controlling the execution are less time critical and will always be performed via the gRPC stack. Hence, no matter if the tensor communication is offloaded to another method, the requirement for setting up communication endpoints stays in place. This offloading functionality is what enables the RDMA over Converged Ethernet (RoCE) support that Google's internal TensorFlow version supports. It also enables (external) contributors to add support for other network stacks, see the next section. Because the changes happen on the deeper levels of TF, the user does not have to make any changes to his designed neural net. The only change required is the specification of the communication protocol in the execution script. However, to take advantage of these contributed packages the user will have to build TF from source and point the build process to the correct libraries. gRPC-based communication can also transparently take advantage of the IPoIB [17] interface if IB is the underlying interconnect.

\section{B. $g R P C+$ ' $X$ ' Designs}

We broadly discuss three gRPC extensions: 1) gRPC+MPI, 2) gRPC+Verbs, and 3) gRPC+GDR.

1) MPI: The gRPC+MPI method adds support for sending tensors using MPI primitives (see Section II-B. Depending on the support that the MPI library offers, this allows TF to make use of advanced network interconnects such as IB and OmniPath. The current implementation of the gRPC+MPI method uses a single thread for all the MPI related operations. On one side, this increases compatibility with MPI frameworks that are not thread-safe, but on the other side, it can hamper performance. Especially when many small data tensors are exchanged there would be enough bandwidth available for parallel transfers which could increase overall performance. MPI processes are uniquely identified using their rank, which is incompatible with the TCP/IP connection strings that gRPC uses to identify processes. Therefore a mapping is created from the gRPC identifiers to the MPI process ranks during the initialization of TF.
2) Verbs: The gRPC+Verbs protocol uses the RDMA-verbs API. By directly using the verbs APIs, there is no need for setting up a global execution environment as required by MPI. However, many features that are available in MPI have to be manually implemented. Examples include features like pinned memory buffers to improve the performance of data-transfers, and the use of GPUDirect RDMA. The pinned memory functionality is implemented in the gRPC+Verbs [3] protocol and can be utilized via the tf.contrib package. Another approach to exploit IB Verbs for gRPC in a more transparent fashion has been presented in [14]. We refer to this as Accelerated gRPC in Figure 1 .

3) GDR: The last contributed protocol is the gRPC+GDR protocol. This protocol adds GPUDirect RDMA (GDR) functionality for NVIDIA GPUs. This allows tensors to be directly $\mathrm{read} / \mathrm{written}$ from/to the GPU memory to/from the network adapter without having to first transfer data to the host memory. This saves a full memory copy and reduces latency. In order to take advantage of this protocol, it is required that the system used has the correct drivers and hardware installed to support this operation. Currently, it is only supported for Intel and AMD CPUs combined with an NVIDIA GPU and Mellanox IB Host Controller Adapter (HCA). The details of this extension are available in [43]. It is pertinent to mention that GDR support has been exploited by MPI runtimes like MVAPICH2 and OpenMPI and can be utilized transparently on systems that support this feature and have the required GPU/HCA hardware available. Unfortunately, gRPC+GDR designs did not run properly on any of our clusters, so no results or discussion is provided for this design.

\section{No-gRPC Designs}

Besides the parameter server method to combine (reduce) the results of worker processes, there is the option to use collective operators to perform the reduction. When using these collective operators, there are no separate processes responsible for gathering and updating the network parameters. Instead, the task is distributed over the worker processes. The collective operator required for these operations is called Allreduce; a communication primitive widely used in the HPC/MPI community. There are two 'No-gRPC' designs available as contributed packages to TF: 1) Baidu and 2) Horovod. Both introduce the so-called reduction operators for TF [21] and exploit the Allreduce primitive. Because the reduction based approach is not available in the standard TF version, it requires a bit more work to make use of it than the protocol changes described above. The way these methods are integrated is by overloading a subset of the default TF operators. During execution, these packages get invoked as part of the network (model) configuration phase and detect which of the parameters used in the execution graph need to be combined. Next, additional reduction operators are inserted into the execution graph. During the execution of the graph, these operators are executed, which invokes the corresponding implementation of the Allreduce operator. A major advantage for the users of these reduction based methods is that the steps 
required to setup the execution of the neural network are much simpler. It is no longer required to launch and configure the additional parameter servers. Furthermore, because both these methods are based on the MPI execution model, the user does not need to configure the endpoints explicitly. Instead, the MPI library/launcher can handle the discovery and connection management.

1) Baidu: Baidu's design is part of the tf.contrib package in the TF codebase. It is called tf.contrib.mpi_collectives and can be enabled during the compilation and configuration steps when building TF from source. This extension is not currently available in the TensorFlow wheels (binary packages) available from Google's website. Baidu's design contains a custom ringreduce implementation of Allreduce built on top of MPI_Send and MPI_Irecv primitives. This gives access to the same MPI advantages as mentioned above, namely extensive network support and support for CUDA-Aware MPI operators which reduces the number of required memory operations.

2) Horovod: The second method, introduced by Uber, is available as an external Python package called Horovod and can be installed using the pip package manager. The design of Horovod is based on the mpi_collectives implementation and as such the user side modifications are similar for these packages. However, Horovod design offers some advantages of Baidu's design. Horovod can take advantage of two different reduction implementations. The first is based on the standard MPI_Allreduce operator. The reduction method that this operator uses depends on the underlying MPI implementation. For example, MVAPICH2-GDR now supports efficient CUDAAware MPI_Allreduce designs as discussed in Section V while default MPICH [2] and OpenMPI only provide naive implementations of MPI_Allreduce for GPU buffers. More specifically, the actual reduction implementation in an MPI runtime can differ based on the supercomputer or cluster that is used. Many system vendors add custom communication methods that are designed and tuned specifically for the specific hardware and network topology. The second Horovod implementation exploits NVIDIA NCCL library, which is based on InfiniBand verbs and CUDA kernels to handle communication across and within nodes, respectively. NCCL2 currently cannot be used on the Piz Daint system because it has the proprietary Aries interconnect, which does not support IB verbs. The MPI variant of Horovod, on the other hand, is portable and can be used on the Piz Daint system via Cray's CUDA-Aware MPI implementation. Horovod also provides a unique feature called "Tensor Fusion". When using this method, several small tensors are combined in a single reduction operation. The idea is that by performing several large reductions instead of many small ones the latency is reduced and bandwidth is used more efficiently. The tensor fusion feature is controlled via a runtime threshold parameter, and we experimentally determine the best threshold for a given platform.

\section{Performance Analysis}

We now present a comprehensive performance comparison of all the aforementioned approaches to perform distributed training using TensorFlow. In order to understand the trends for distributed training, the most fundamental question that often gets missed is: What is the best batch size one should use for distributed training?. In this context, the general intuition is that a bigger batch size will lead to better performance. However, we perform single-GPU experiments to understand this behavior in a more pragmatic fashion. Figure 2 shows the impact of the batch size for three GPU generations: 1) Kepler (K80), 2) Pascal (P100), and 3) Volta (V100). The key insight here is: faster GPUs offer better performance for larger batch sizes up to the point of diminishing returns. For ResNet-50, the sweet spot seems to be 64 for all three GPUs. Thus, we choose 64 as the single-GPU batch-size baseline and utilize this for all distributed training experiments performed for ResNet-50. The performance numbers for all

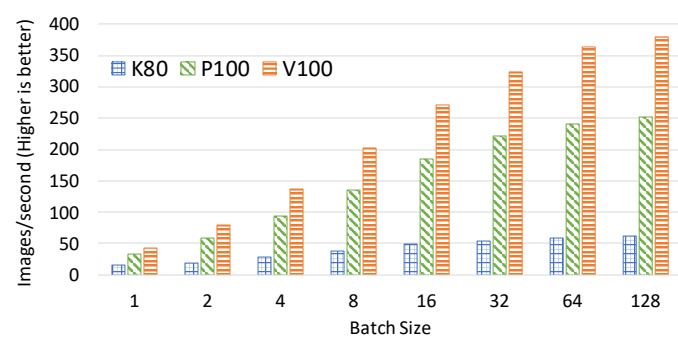

Fig. 2. Effect of Batch Size on Performance for Different GPU Architectures

six approaches except grpc+gdr are presented in Figure 3 The main insight we gain from these experiments is: $g R P C$ and grpc+ ' $X$ ' designs are, in general, slower than Horovod designs. Secondary observations include: 1) Baidu's design despite using the ring-allreduce lags behind Horovod-NCCL and grpc+ ' $\mathrm{X}$ ' designs and 2) Horovod-MV2 is always slower than Horovod-NCCL2. We have used TensorFlow version 1.10.0 for all our experiments. Performance of Allreduce: The observation about the different performance we get for NCCL2 compared to MVAPICH2 for Horovod designs merits further investigation. This is because the only difference in Horovod-NCCL and Horovod-MPI design is the utilization of the corresponding Allreduce primitive. Thus, we perform additional experiments using MPI/NCCL benchmarks to better understand this behavior. Indeed, the performance of NCCL Allreduce is better than MPI_Allreduce in MVAPICH2, as shown in Figure 4 . We performed a thorough analysis of Allreduce designs in the MVAPICH2 library and found a clear opportunity for performance optimizations to deal with DL workloads (large message sizes). Based on this, we propose optimizations for Allreduce and highlight the performance benefits we observed for these enhancements in Section $\nabla$

\section{OPTIMIZING tf_cnn_benchmarks FOR HPC SYSTEMS}

The performance analysis presented in Section III was made possible via the official TensorFlow benchmarking scripts 


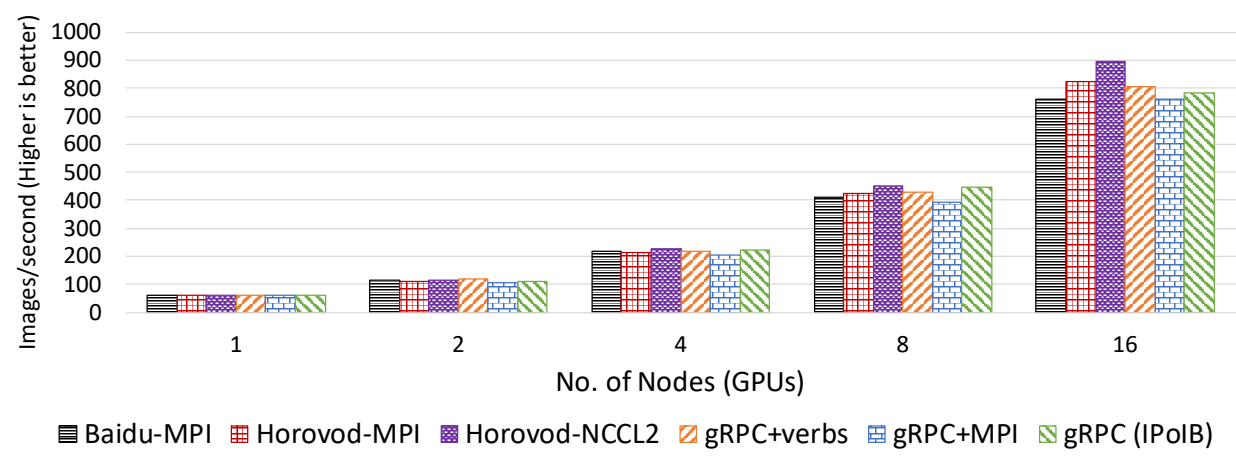

Fig. 3. Performance Analysis of Six TensorFlow Approaches for Distributed Training of ResNet-50 on the RI2 Cluster (see Section VI-B). 1) gRPC was executed using IP addresses of the InfiniBand interface to make sure that tensor communication happens using the efficient IPoIB channel. 2) MPI refers to the MVAPICH2 [30] MPI library for all cases. 3) The latest version of NCCL2 (2.3.4) was used for all the experiments.

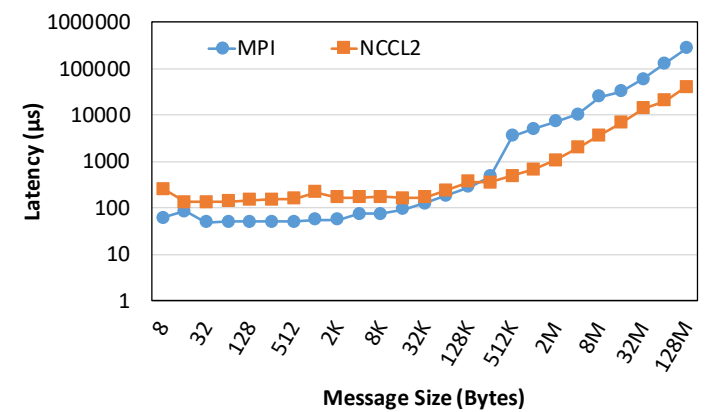

Fig. 4. Micro-benchmark Performance for MPI_Allreduce and NCCL Allreduce on the RI2 Cluster (see Section VI-B. 1) MPI refers to existing MVAPICH2 MPI Library and 2) NCCL2 refers to NCCL 2.3.4

called tf_cnn_benchmarks [20]. To evaluate various platforms and distributed TF implementations, we modified these application level benchmarks and added support for running Baidu's design. We also modified the scripts to enable gRPC and $\mathrm{gRPC}+\mathrm{X}$ runs using SLURM [44]. During the startup phase, we pull in the SLURM environment variables in order to determine the total number of launched benchmark instances and their unique IDs (rank). This is required to setup the configurations that do not use MPI, but also require that each process is uniquely identified. The unique ID is consequently used to determine the type of process (worker, or parameter server) and to setup the underlying network connections. Because this is based on the SLURM environment variables it is trivial to adapt this to other workload managers, by using the variables that are specific to that workload manager or particular mpirun version. Once this setup is complete, and the various processes are connected to each other, the neural network will be initialized. This modification to tf_cnn_benchmarks allowed us to test all of the several distributed training configurations with exactly the same neural network design and data processing routines. This ensures that any performance differences between the various networking options can be directly attributed to the reduction algorithm and the networking library. The benchmark suite allows users to test the performance of various convolutional neural networks using TensorFlow. For our tests, we use three different image classification networks, MobileNet [23], ResNet-50 [22] and NASNet-large [45]. To prevent that our results are influenced by file I/O (disk) performance, we only use synthetic input data. The training phase for all the various approaches will remain common and contains exactly the same operations as the real neural network training with the difference being the usage of synthetic data instead of real images that need to be read from a storage subsystem. After a number of warm-up iterations, a set of ten iterations will be performed to determine the image throughput rate. Note that because synthetic data is used we purely measure the GPU and network performance for the multi-node cases but only the GPU performance for a single process case. The CPU (used for decoding image data) and storage media (used for loading data) are not part of our tests since we focus only on the scaling characteristics of distributed training. However, when doing real training runs these components will influence the final performance, especially the storage media will be important as a large number of GPUs will have to be fed with fresh training data during each iteration.

\section{OPTIMIZING CUDA-AWARE MPI PRIMITIVES}

Based on the analysis in Section III. we observed that the MPI_Allreduce primitive is a significant performance bottleneck for the Horovod-MPI approach. Thus, the primary challenge we have to deal with is a better and more efficient MPI_Allreduce design to tackle DL workloads at scale. To address this, we propose two major design optimizations: A) Truly CUDA-Aware reduction method that exploits CUDA based computations in addition to GPUDirect-based communication, and B) An advanced caching scheme for GPU-based (device) pointers to avoid query overhead in the critical path.

\section{A. CUDA Kernel-enabled Allreduce for large messages}

Although offloading reduction operations to GPU kernels has been discussed before [16], [27], [33], for large messages it has not fully leveraged the GPU computing power for Allreduce algorithms. To perform large-message Allreduce operations efficiently, a variety of "reduce-scatter followed by allgather (RSA)" algorithms are widely used. Two popular 
algorithms are: 1) Ring-based RSA [34]: first a virtual ring is constructed between processes. Next, each process sends a chunk of data to its left neighbor and performs reduction upon the chunk received from its right neighbor for $(p-1)$ steps, where $p$ denotes the number of processes. At the end of this reduce-scatter phase, each process owns a partial final result vector. Then a similar ring-based allgather is performed to gather the complete final result (notable implementations: NCCL and Baidu Allreduce). 2) Recursive vector halving and doubling RSA [41]: each process pair exchanges half of the vector/message and reduces it. Next, the message size is halved, and a different pair of processes is selected for $\log p$ steps. Allgather is performed in reverse and doubles the message size at each of the $\log p$ steps (notable implementations MPICH and MVAPICH2). The first method is popular because of the bandwidth-optimized feature, while the second method is expected to be more efficient at scale. However, the existing MVAPICH2 implementation of recursive vector halving and doubling RSA relies on the CPU to perform reduction operations, which is a waste of GPU compute power. In this paper, we propose and implement a CUDA-Aware MPI_Allreduce primitive using recursive vector halving and doubling RSA, with GPU kernel-enabled reduction operations. There are three major benefits: 1) significantly reduces the reduction latency for large messages by fully utilizing the massive parallelism and high-bandwidth memory of GPUs. 2) Avoids expensive data movement from GPU to host memory. 3) Leverages advanced hardware and software features such as GPUDirect RDMA to improve intra-node and inter-node communication.

\section{B. Optimized Pointer Cache for Device Buffers}

The CUDA unified addressing feature allows NVIDIA GPU devices to share a unified address space with the host. This means that there is no distinction between a device pointer and a host pointer, i.e., the same pointer value could represent host memory or device memory at different times. MPI libraries like MVAPICH2 have implemented various algorithms to optimize MPI primitives for host and device buffers. Therefore, the MPI runtime needs to identify the buffer type before selecting the most performant algorithm. The CUDA driver provides a low-level API to perform this identification. However, each MPI call may need to query the buffer type multiple time even when the pointer value remains unchanged. This incurs a significant delay in the MPI function as the call accesses multiple driver modules as illustrated in Figure 5 (red dashed arrow). To mitigate this overhead, we designed a Pointer Cache, which stores the pointer information, as depicted in Figure 5 (dark-red solid box and arrows). There are two ways to maintain the cache: 1) one-time driver lookup at MPI-level, 2) interception of the device allocation APIs at application-level. In the first approach, the MPI runtime caches (i.e., inserts) the buffer type of the given pointer when it is seen for the first time. However, the runtime is not able to invalidate a cache entry when the buffer gets de-allocated by the application without notifying the MPI runtime. This leads

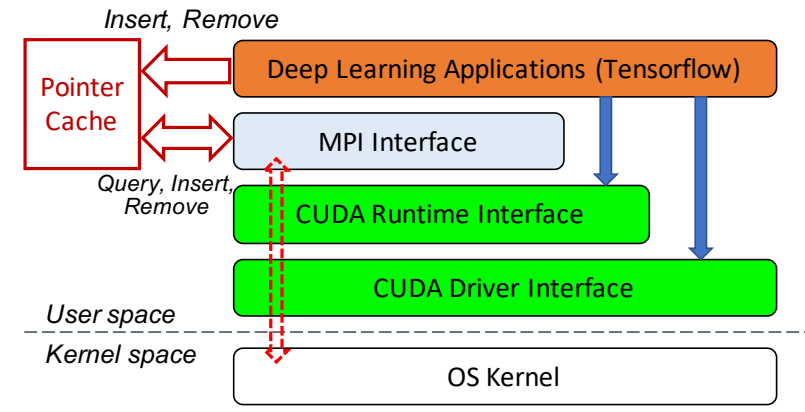

Fig. 5. Pointer Cache Design (dark-red box and arrows) to avoid expensive queries to the CUDA driver (red dashed arrow).

to the second approach. We let the MPI runtime intercept CUDA memory management APIs, e.g., cuMalloc and cuFree, and update the pointer cache accordingly. This way, the MPI runtime only has to query the cache but not maintain it. This optimization improves the performance of all CUDA-aware MPI primitives, including the ones primarily used by the deep learning frameworks.

\section{Performance Benefits}

To evaluate the proposed optimizations, we conducted experiments across 16 NVIDIA Tesla K80 GPU nodes at a local cluster (RI2). We used micro-benchmarks to compare the Allreduce performance between default MVAPICH2 (MPI), NVIDIA NCCL (NCCL2), and the new MVAPICH2 implementation (MPI-Opt). Figure 6 shows that the new method yields $4.1 \times$ faster Allreduce compared to the default MVAPICH2 solution for small and medium messages (i.e., smaller than $128 \mathrm{~KB}$ ) due to the pointer cache. Yet, MPI-Opt is $17 \times$ faster than NCCL2 for Allreduce operation of 8-byte data. For larger message sizes, the optimized GPU kernel-enabled reduction scheme provides up to $8 \times(1.4 \times)$ performance improvement compared to the default MVAPICH2 (NCCL2) library. With the significant benchmark-level improvements, we next present the application level performance of the enhanced MPI primitives.

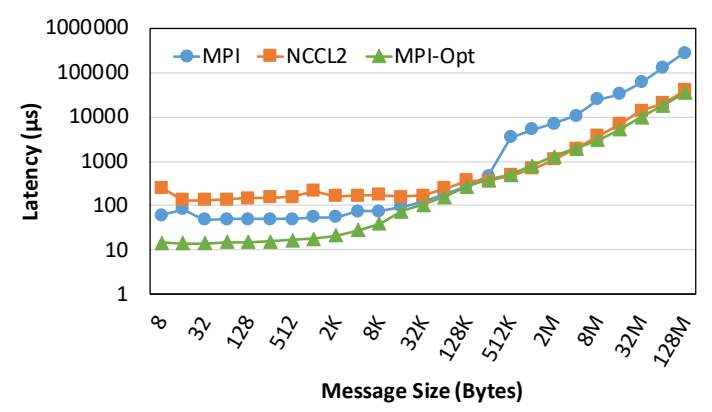

Fig. 6. Benefits of the Allreduce optimizations. 1) MPI refers to existing MVAPICH2 MPI Library. 2) MPI-Opt refers to the optimizations made available in MVAPICH2-GDR 2.3rc1 


\section{Vi. Comprehensive Performance Comparison of EXISTING AND PROPOSED DESigns}

We now present an in-depth performance comparison of all the distributed training approaches with existing communication libraries as well as with our proposed Allreduce design schemes. We perform various experiments on three different clusters: 1) The RI2 cluster at The Ohio State University, 2) The Owens cluster at Ohio Supercomputing Center (OSC), and 3) The Piz Daint cluster at Swiss National Supercomputing Centre.

\section{A. Performance of CPU vs. GPU based Training}

We performed several GPU and CPU based training experiments. However, there are many additional vectors that need to be considered to provide a sound performance evaluation. The default CPU installation of TensorFlow offers much slower performance compared to GPU-based training. We also explored the Intel-optimized TensorFlow version. However, we chose to only focus on GPU results in this paper as the discussion and comparison of default CPU, optimized CPU, and GPU based training is beyond the scope of this paper.

\section{B. $R I 2$}

The RI2 cluster at The Ohio State University consists of 20 nodes connected via Mellanox SB7790 and SB7800 InfiniBand switches. Each node is equipped with two 14-core Intel (Broadwell) Xeon E5-2680 v4 $2.4 \mathrm{GHz}$ processors, 128 GB DDR3 Memory, one NVIDIA Tesla K80 GPU, and one single port InfiniBand EDR HCA. Based on the proposed optimizations for Allreduce discussed in Section $\mathrm{V}$, we now provide a comparison of the best performing Horovod-NCCL approach (shown earlier in Figure 3), Horovod-MPI, and the proposed Horovod-MPI-Opt approach that takes advantage of the optimized MPI_Allreduce design. As we can clearly see in Figure 7. Horovod-MPI-Opt performs much better than Horovod-MPI as well as offering better/comparable performance as Horovod-NCCL2 for most cases. The "ideal" bar for all the graphs in this section has been calculated using a linear speedup formula: Ideal = Images $/$ second (for 1 GPU) $\times$ \#GPUs

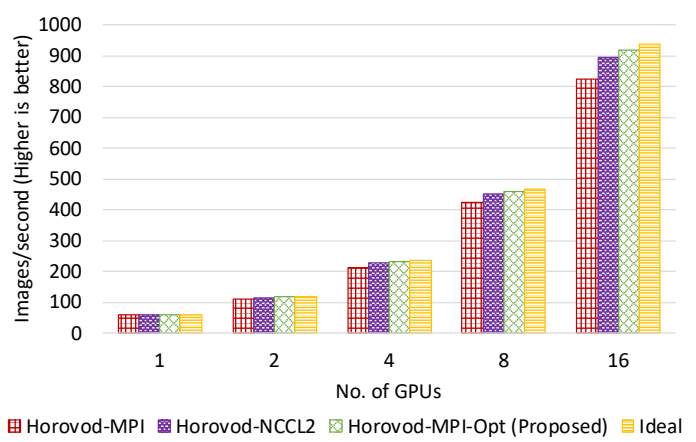

Fig. 7. Performance comparison for ResNet-50 training using three Horovod versions on the RI2 Cluster (up to 16 GPUs). 1) NCCL 2.3.4 was used for NCCL experiments. 2) MPI refers to the MVAPICH2 [30] library. 3) MPI-Opt refers to the new Allreduce designs made available as part of the MVAPICH2GDR 2.3rc1 library.

\section{Owens}

Owens is a 23,392-core Dell Intel Xeon E5-2680 v4 machine with $160 \mathrm{GPU}$ nodes equipped with NVIDIA Pascal P100 GPUs. Each node is equipped with a dual-socket Intel Xeon processor (28 cores) and an IB EDR HCA. We have used the Owens cluster to extend our finding from RI2 to a larger scale as well as a newer GPU generation. The cluster is heavily used by HPC researchers across the US, so we limit our performance evaluation to only the best performing TF variants from RI2. Figure 8 provides the performance comparison of Horovod-NCCL2 and Horovod-MPI-Opt for the distributed training of ResNet-50 on up to 64 Pascal GPUs. Clearly, we can observe that our proposed Allreduce optimizations have enabled Horovod-MPI-Opt to achieve near-ideal scaling that is better/comparable to Horovod-NCCL designs. It is pertinent to mention that the Horovod designs have been the best for all configurations on RI2 as well as the Owens cluster.

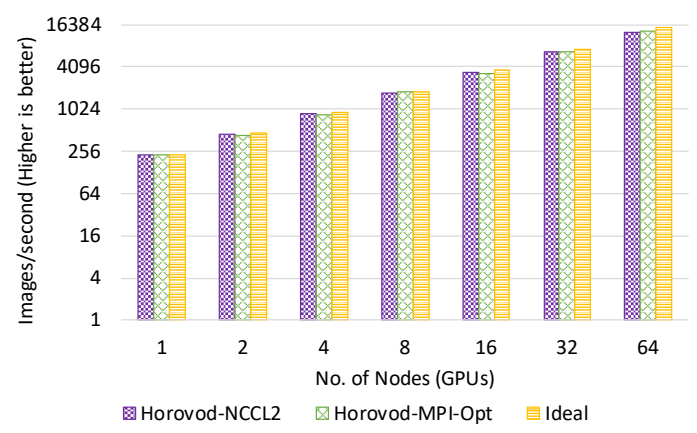

Fig. 8. Performance comparison for ResNet-50: Training performed using two Horovod designs on the Owens Cluster (up to 64 GPUs). 1) NCCL 2.3.4 was used for NCCL experiments. 2) Horovod-MPI-Opt refers to the design that takes advantage of the new Allreduce implementation made available in the MVAPICH2-GDR 2.3rc1 library.

\section{Piz Daint}

We now provide the scaling results up to an even larger scale (up to 128 Pascal GPUs) on the Piz Daint cluster. We also extend our evaluation for Piz Daint with two more DNN architectures: 1) MobileNet [23] and 2) NASNet [45]. Each node of the Piz Daint cluster is equipped with a single P100 GPU and a 12-core Intel Xeon E5-2690 v3 (Haswell) CPU. The nodes are connected using the Cray Aries communication technology based on the Dragonfly topology. The machine has thousands of compute nodes so the actual placement (distance) between the processes is random and can influence the actual execution time. The software stack is designed by Cray and optimized for the machine. This means that we have limited control over the used (MPI) libraries and just use what the system has available. Because of this software limitation, we were not able to test out the Horovod-NCCL implementation on this system as there is no support for IB verbs, which NCCL uses for inter-node communication. For Horovod-MPI, we have used the default Cray-MPICH (v7.6) library. The scaling results for Piz Daint are presented in Figure 9 for four different approaches (Horovod-MPI, gRPC, gRPC+MPI, and HorovodMPI). It is clear that gRPC+MPI approach shows the worst 
scaling. This is because of its single-threaded implementation. The bottleneck is especially visible for NASNet-large model as a large number of model parameters need to be transferred over the network. Baidu-MPI and Horovod-MPI perform very similar, with Horovod-MPI showing slightly better results. The gRPC results fall slightly behind the Baidu-MPI and HorovodMPI solutions. Given that all these implementations use the same underlying communication network the performance difference can be attributed to the way the parameter server solution is implemented. While training approaches have an impact on performance, the scaling behavior is also directly related to the size of the DNN. Mobilenet shows worst scaling compared to the ideal (16\% efficiency for Horovod-MPI), as communication of gradients cannot be overlapped (hidden behind) the relatively smaller computation. NASNet-large, the largest network we have tested, shows near-ideal scaling (92\% efficiency for Horovod-MPI as the computation is large enough to be overlapped with gradient communication. Resnet-50 falls in between the two extremes with $71 \%$ efficiency for HorovodMPI.

\section{RELATED WORK}

There are a significant number of research efforts from academia and industry toward understanding and developing scalable deep learning systems. Ben-Nun and Hoefler present an in-depth concurrency analysis of various DNN architectures with a focus on distributed training in [13]. Cui et al. propose a "GeePS" parameter server to support scalable data-parallel training across GPU nodes by mitigating data movement overhead caused by GPU stalling in [18]. Chilimbi et al. propose a distributed system called Adam to achieve scalable DL training for large DNN models, and a 120-machine cluster was used for a demonstration [15]. Shi et al. evaluated the performance of distributed DL frameworks such as TensorFlow, CNTK, Caffe-MPI and MXNet over single-GPU, multi-GPU, and multi-node (4 nodes) scenarios [38], [39]. They identify several performance bottlenecks such as I/O access and data communication among GPUs. However, the scalability of these DL frameworks is not evaluated. The authors of [40] have presented an extension of the Horovod [37] design that uses the Intel Machine Learning Scaling (MLSL) library for distributed training with TensorFlow. However, the study is focused on scaling CPU-based training only and no GPU results are presented.

\section{CONCLUSION}

In this work, we evaluated a wide range of communication methods that can be used with the TensorFlow deep learning framework. Our focus has been on the scalability, and usability of the various techniques. We compared MPI based reduction methods, custom written reduction schemes, and TensorFlow's native parameter server model. For our evaluation, we used three different image classification networks, each with a significantly different number of parameters to be exchanged. We further introduced an improved reduction method for GPU based data. The optimized designs have been integrated into
MVAPICH2 and have been made publicly available since the MVAPICH2-GDR 2.3rc1 release. This method shows up to $1.4 \times$ faster Allreduce operations compared to the NVIDIA NCCL2 communication library in micro-benchmarks. Our performance evaluation shows that the customized reduction based methods, i.e., Horovod-MPI and Horovod-NCCL, outperform the parameter server methods, i.e., gRPC and $\mathrm{gRPC}+\mathrm{X}$, for all three evaluated neural nets and all three systems. The experimental results show that the proposed optimizations help Horovod-MPI achieve approximately 98\% and $90 \%$ scaling efficiency for training on 16 and 64 GPU nodes on the RI2 and Owens clusters, respectively. Finally, HorovodMPI yields $1.8 \times$ and $3.2 \times$ higher throughput than the native gRPC method for ResNet-50 and MobileNet, respectively, on the Piz Daint cluster across 128 nodes.

\section{ACKNOWLEDGMENT}

This research is supported in part by National Science Foundation grants \#CNS-1513120, \#ACI-1450440 and \#CCF1565414, and a grant from the Swiss National Supercomputing Centre (CSCS) under project ID s716. The authors would like to thank Jonathan Perkins and Dr. Khaled Hamidouche for extending invaluable help in implementing the pointer cache design for MVAPICH2-GDR.

\section{REFERENCES}

[1] "ImageNet," http://image-net.org/ [Online; accessed October 29, 2018].

[2] "MPICH: High-Performance Portable MPI," https://www.mpich.org/, [Online; accessed October 29, 2018].

[3] "Open Sourcing TensorFlowOnSpark: Distributed Deep Learning on Big-Data Clusters," http://yahoohadoop.tumblr.com/post/157196317141/open-sourcingtensorflowonspark-distributed-deep [Online; accessed October 29, 2018].

[4] "Protocol Buffers," https://developers.google.com/protocol-buffers/, [Online; accessed October 29, 2018].

[5] "PyTorch," https://pytorch.org/, [Online; accessed October 29, 2018].

[6] "Tensorflow: An Open Source Machine Learning Framework for Everyone," https://github.com/tensorflow/tensorflow, [Online; accessed October 29, 2018].

[7] "UCX-Unified Communication X," http://www.openucx.org/ [Online; accessed October 29, 2018].

[8] "Intel/caffe," https://github.com/intel/caffe, 2016, [Online; accessed October 29, 2018].

[9] "Caffe2 Framework," https://caffe2.ai 2017.

[10] "Gloo: Collective Communication Library with Various Primitives for Multi-machine Training." https://github.com/facebookincubator/gloo, 2017, [Online; accessed October 29, 2018].

[11] M. Abadi, P. Barham, J. Chen et al., "Tensorflow: A system for largescale machine learning," in 12th USENIX Symposium on Operating Systems Design and Implementation (OSDI 16). USENIX Association, 2016, pp. 265-283.

[12] A. A. Awan, K. Hamidouche, J. M. Hashmi, and D. K. Panda, "S-Caffe: Co-designing MPI Runtimes and Caffe for Scalable Deep Learning on Modern GPU Clusters," in Proceedings of the 22nd ACM SIGPLAN Symposium on Principles and Practice of Parallel Programming, ser. PPoPP '17. New York, NY, USA: ACM, 2017.

[13] T. Ben-Nun and T. Hoefler, "Demystifying Parallel and Distributed Deep Learning: An In-Depth Concurrency Analysis," CoRR, vol. abs/1802.09941, Feb. 2018.

[14] R. Biswas, X. Lu, and D. K. Panda, "Accelerating TensorFlow with Adaptive RDMA-based gRPC," in 25th IEEE International Conference on High Performance Computing, Data, and Analytics (HiPC'18), December 2018. 


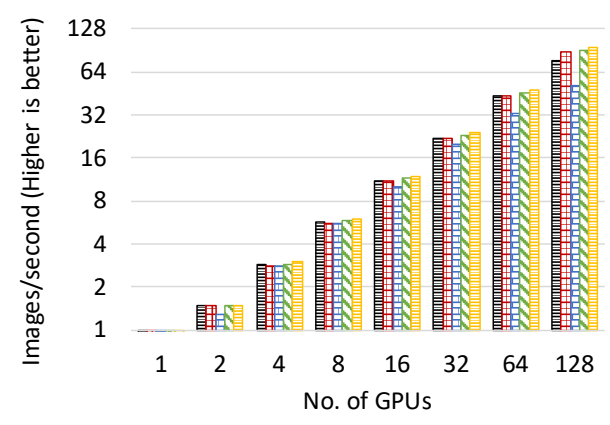

(a) NASNet-large

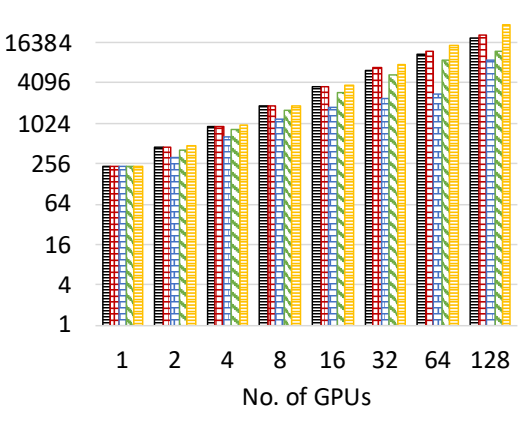

EBaidu-MPI $\boxplus$ Horovod-MPI $\square$ gRPC+MPI $₫$ gRPC Eideal

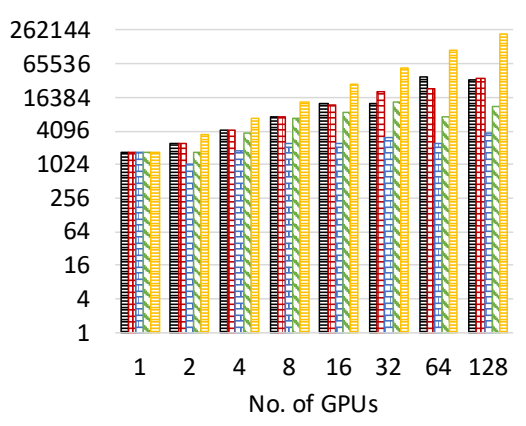

(c) Mobilenet

Fig. 9. Performance Comparison for Various Distributed Training Approaches using 1) NASNet-large, 2) ResNet-50, and 3) Mobilenet on the Piz Daint Cluster (up to 128 GPUs). MPI refers to Cray-MPICH (v7.6) MPI Library.

[15] T. Chilimbi, Y. Suzue, J. Apacible, and K. Kalyanaraman, "Project Adam: Building an Efficient and Scalable Deep Learning Training System," in 11th USENIX Symposium on Operating Systems Design and Implementation (OSDI 14). Broomfield, CO: USENIX Association, 2014, pp. 571-582.

[16] C. Chu, K. Hamidouche, A. Venkatesh, A. A. Awan, and D. K. Panda "CUDA Kernel Based Collective Reduction Operations on Large-scale GPU Clusters," in 2016 16th IEEE/ACM International Symposium on Cluster, Cloud and Grid Computing (CCGrid), May 2016, pp. 726-735.

[17] J. Chu and V. Kashyap, "Transmission of IP over InfiniBand (IPoIB)," Requests for Comments, RFC Editor, RFC 4391, Apr 2006. [Online]. Available: https://tools.ietf.org/html/rfc4391

[18] H. Cui, H. Zhang, G. R. Ganger, P. B. Gibbons, and E. P. Xing, "GeePS: Scalable Deep Learning on Distributed GPUs with a GPU-specialized Parameter Server," in Proceedings of the Eleventh European Conference on Computer Systems, ser. EuroSys '16. New York, NY, USA: ACM, 2016, pp. 4:1-4:16.

[19] Google, "Google's Remote Procedure Call Library (gRPC)," http://www. grpc.io

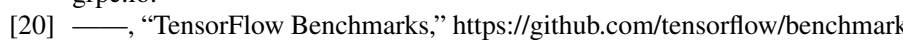
2018, [Online; accessed Oct-2018].

[21] —, "TensorFlow Custom Operators: Adding an Op," https://www.tensorflow.org/extend/adding_an_op, 2018, [Online; accessed Oct-2018].

[22] K. He, X. Zhang, S. Ren, and J. Sun, "Deep Residual Learning for Image Recognition," ArXiv e-prints, Dec. 2015.

[23] A. G. Howard, M. Zhu, B. Chen, D. Kalenichenko, W. Wang, T. Weyand, M. Andreetto, and H. Adam, "Mobilenets: Efficient convolutional neural networks for mobile vision applications," CoRR, vol. abs/1704.04861, 2017.

[24] F. N. Iandola, M. W. Moskewicz, K. Ashraf, and K. Keutzer, "FireCaffe: Near-linear Acceleration of Deep Neural Network Training on Compute Clusters," in Proceedings of the IEEE Conference on Computer Vision and Pattern Recognition, 2016, pp. 2592-2600.

[25] Y. Jia, E. Shelhamer, J. Donahue, S. Karayev, J. Long, R. Girshick, S. Guadarrama, and T. Darrell, "Caffe: Convolutional Architecture for Fast Feature Embedding," arXiv preprint arXiv:1408.5093. 2014.

[26] M. Li, D. G. Andersen, J. W. Park, A. J. Smola, A. Ahmed, V. Josifovski, J. Long, E. J. Shekita, and B.-Y. Su, "Scaling distributed machine learning with the parameter server," in Proceedings of the 11th USENIX Conference on Operating Systems Design and Implementation, ser. OSDI'14. Berkeley, CA, USA: USENIX Association, 2014, pp. 583-598. [Online]. Available: http://dl.acm.org/citation.cfm?id= 2685048.2685095

[27] X. Luo, W. Wu, G. Bosilca, T. Patinyasakdikul, L. Wang, and J. Dongarra, "ADAPT: An Event-based Adaptive Collective Communication Framework," in Proceedings of the 27th International Symposium on High-Performance Parallel and Distributed Computing, ser. HPDC '18. New York, NY, USA: ACM, 2018, pp. 118-130.

[28] H. Meuer, E. Strohmaier, J. Dongarra, and H. Simon, "TOP 500 Supercomputer Sites," http://www.top500.org
[29] Microsoft, “CNTK," http://www.cntk.ai/ 2017, [Online; accessed April2017].

[30] MVAPICH2: High Performance MPI over InfiniBand and iWARP, http://mvapich.cse.ohio-state.edu/] [Online; accessed October 29, 2018]

[31] NVIDIA, "AI Research \& Developement - NVIDIA DGX Systems," https://www.nvidia.com/en-us/data-center/dgx-systems/, [Online; accessed October 29, 2018].

[32] NVIDIA, "NCCL," https://github.com/NVIDIA/nccl, 2017, [Online; accessed October 29, 2018].

[33] L. Oden, B. Klenk, and H. Frning, "Energy-Efficient Collective Reduce and Allreduce Operations on Distributed GPUs," in 2014 14th IEEE/ACM International Symposium on Cluster, Cloud and Grid Computing, May 2014, pp. 483-492.

[34] P. Patarasuk and X. Yuan, "Bandwidth Optimal All-reduce Algorithms for Clusters of Workstations," J. Parallel Distrib. Comput., vol. 69, no. 2, pp. 117-124, Feb. 2009.

[35] H. Pritchard, D. Gilmore, M. ten Bruggencate, D. Knaak, and M. Pagel, "Message passing toolkit (MPT) software on XT3," in Cray User Group 2006 Proceedings, 2006.

[36] D. E. Rumelhart, G. E. Hinton, and R. J. Williams, "Parallel Distributed Processing: Explorations in the Microstructure of Cognition, Vol. 1," D. E. Rumelhart, J. L. McClelland, and C. PDP Research Group, Eds. Cambridge, MA, USA: MIT Press, 1986, ch. Learning Internal Representations by Error Propagation, pp. 318-362.

[37] A. Sergeev and M. D. Balso, "Horovod: Fast and Easy Distributed Deep Learning in TensorFlow," CoRR, vol. abs/1802.05799, 2018.

[38] S. Shi and X. Chu, "Performance Modeling and Evaluation of Distributed Deep Learning Frameworks on GPUs," CoRR, vol. abs/1711.05979, 2017.

[39] S. Shi, Q. Wang, P. Xu, and X. Chu, "Benchmarking State-of-the-Art Deep Learning Software Tools," CoRR, vol. abs/1608.07249, 2016.

[40] S. Sridharan, K. Vaidyanathan, D. Kalamkar, D. Das, M. E. Smorkalov, M. Shiryaev, D. Mudigere, N. Mellempudi, S. Avancha, B. Kaul, and P. Dubey, "On Scale-out Deep Learning Training for Cloud and HPC," ArXiv e-prints, Jan. 2018

[41] R. Thakur, R. Rabenseifner, and W. Gropp, "Optimization of Collective Communication Operations in MPICH," Int. J. High Perform. Comput. Appl., vol. 19, no. 1, pp. 49-66, Feb. 2005.

[42] The Open MPI Development Team, "Open MPI : Open Source High Performance Computing," http://www.open-mpi.org

[43] B. Yi, J. Xia, L. Chen, and K. Chen, "Towards Zero Copy Dataflows Using RDMA," in Proceedings of the SIGCOMM Posters and Demos, ser. SIGCOMM Posters and Demos '17. New York, NY, USA: ACM, 2017, pp. 28-30.

[44] A. B. Yoo, M. A. Jette, and M. Grondona, "SLURM: Simple Linux Utility for Resource Management," in JSSPP 2003. Springer, 2003, pp. 44-60.

[45] B. Zoph, V. Vasudevan, J. Shlens, and Q. V. Le, "Learning Transferable Architectures for Scalable Image Recognition," CoRR, vol. abs/1707.07012, 2017. 